\title{
EVALUACIÓN FENOLÓGICA Y DIGESTIBILIDAD IN VIVO DE LA LEGUMINOSA FORRAJERA (Arachis pintoi) EN DIFERENTES EDADES DE CORTE
}

\author{
PHENOLOGICAL EVALUATION AND IN VIVO DIGESTIBILITY OF THE FORAGE LEGUME \\ (Arachis pintoi) CUT AT DIFFERENT AGES
}

\author{
${ }^{\bullet}$ Víctor Godoy Espinoza ${ }^{1}$, Alexandra Barrera Alvarez ${ }^{1,2}$, Roque Vivas Moreira ${ }^{1}$, Jorge Quintana Zamora ${ }^{1,2}$, \\ Mayra Peña Galeas ${ }^{1,2}$, Leoncio Villota González ${ }^{3}$, Lola Casanova Ferrín ${ }^{2}$, Juan Avellaneda Cevallos ${ }^{1,2}$

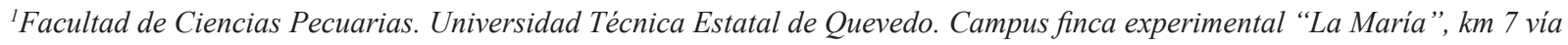 \\ Quevedo-El Empalme. C. P. 73. Mocache,Los Ríos, Ecuador. ${ }^{\circledR} v i \_g o d o y @ h o t m a i l . c o m$ \\ ${ }^{2}$ Dirección de Investigación Cientifica y Tecnológica. Universidad Técnica Estatal de Quevedo. Campus finca experimental "La \\ Maria”, km 7 vía Quevedo-El Empalme. C. P. 73. Mocache, Los Ríos, Ecuador. \\ ${ }^{3}$ Facultad de Ciencias Agropecuarias, Carrera de Ingeniería Zootécnica. Universidad Técnica de Esmeraldas Luis Vargas \\ Torres. Parroquia San Mateo, Estación Experimental Mutile. Esmeraldas, Esmeraldas, Ecuador.
}

\begin{abstract}
RESUMEN
$E_{\text {tis }}^{1}$ objetivo de ésta investigación fue evaluar la fenología, producción, composición química nutricional y digestibilidad in vivo de $A$. pintoi y de establecer modelos matemáticos para determinar el valor nutricional y producción en base a la composición química de la planta. Las evaluaciones fenológicas se realizaron en la hacienda ESPE y la digestibilidad in vivo en la Facultad de Ciencias Pecuarias de la ESPOCH. En la primera fase se utilizaron parcelas experimentales de $10 \times 15 \mathrm{~m}$ con cuatro repeticiones, mientras que en la segunda fase la unidad experimental fue un ovino $(n=4)$. En ambos ensayos se aplicó un diseño experimental completamente al azar con un modelo lineal aditivo utilizando un nivel de significancia del 5\%. Se realizó un análisis de correlación y regresión lineal entre variables fenológicas, composición química, digestibilidad y energía. Todos los análisis fueron realizados con el paquete SPSS 10. Las mayores producciones de forraje verde y materia seca (MS) por hectárea se obtuvieron con cortes a los 75 días con 47,730 y 12,480 kg ha-1, respectivamente. En cuanto a la composición química de la proteína bruta y digestibilidad in vivo de la MS, el forraje cortado a 30 días obtuvo los mayores valores con 24.50 y $66.42 \%$, respectivamente. El máximo valor de energía neta de lactancia $\left(\mathrm{EN}_{\mathrm{L}}\right)$ se logró en el corte de 30 días, alcanzando 1.51 para luego disminuir a 1.24 Mcal $\mathrm{EN}_{\mathrm{L}} \mathrm{kg}^{-1}$ a los 75 días.
\end{abstract}

Palabras clave: Leguminosa forrajera, digestibilidad in vivo, maní.

\footnotetext{
Recibido: 29-Marzo-2012. Recibido en forma corregida: 28-Mayo-2012. Aceptado: 20-Noviembre-2012.

Publicado como ARTÍCULO CIENTÍFICO en Ciencia y Tecnología 5(2): 7-16. 2012
}

\begin{abstract}
$T$ he objective in this research was to evaluate the phenology, production and to determine nutritional chemistry composition and in vivo digestibility of $A$. pintoi and, to establish standards for determining the nutritional value and production based according to the chemistry composition of the plant. The phenological evaluations were carried out at ranch ESPE and in vivo digestibility was executed in Animal Science Faculty from ESPOCH. In the first phase were used experimental plots 10 by $15 \mathrm{~m}$. in four replications. In the second phase the experimental unit was an ovine $(n=4)$. In both tests were used at random experimental designs with a linear additive standard applying a significance level of $5 \%$. It was executed an correlation analysis and linear regression among phonological variables, chemistry composition, digestibility and energy. The whole analysis were carried out with SPSS 10 Statistical package. The greatest productions of green fodder and dry matter $(\mathrm{DM})$ per hectare were obtained at cutting to 75 days with 47,730 and $12,480 \mathrm{~kg} \mathrm{ha}^{-1}$, respectively. On the other hand, in chemistry composition of grass protein and in vivo digestibility of dry Matter (DM), the clipped forage to 30 days obtained the greatest values with 24.50 and $66.42 \%$, respectively. The maximum value of net energy for lactation $\left(\mathrm{NE}_{\mathrm{L}}\right)$ was achieved at 30 days cutting reaching 1.51 for decreasing to 1.24 Mcal $\mathrm{NE}_{\mathrm{L}} \mathrm{kg}^{-1}$ to 75 days.
\end{abstract}

Key words: Forage legume, in vivo digestibility, peanut. 


\section{INTRODUCCIÓN}

$\mathrm{D}$ e acuerdo con los datos del censo SICA/MAG (2002), las tierras agropecuarias del Ecuador en 1999-2000 alcanzaban a 12,400,000 hectáreas, $27 \%$ de las cuales estaban bajo pasturas sembradas, 9.1\% bajo pasturas nativas, $4.9 \%$ cubierta por páramos y $3 \%$ bajo barbecho. El área de pasturas sembradas, nativas y naturalizadas en Ecuador ha sido estimada en 5,510,000 hectáreas (SICA/MAG, 2002). Si todos estos elementos son considerados recursos pastoriles, cerca de la mitad de la tierra utilizable estaba disponible para pastoreo.

Los sistemas tradicionales de producción animal bovina en el trópico ecuatoriano están basados generalmente en el uso de gramíneas autóctonas como especies del género pennisetum o introducidas como el Kikuyo o pasto elefante, más la utilización deficiente de leguminosas forrajeras como las del género Crotalaria, Desmodium o especies arbóreas de Leucaena (Acosta, 1997). Estos aspectos se manifiestan en los esquemas deficitarios de la alimentación básica de los rumiantes. Investigaciones en alimentos alternativos, como la utilización de subproductos del procesamiento de la fruta, han demostrado ser una alternativa nutritiva para los animales en épocas de sequía, así como también el maní forrajero, A. pintoi, el cual es originario de América del Sur restringido naturalmente a Brasil, Paraguay, Argentina y Uruguay (Valls y Simpson, 1995). Este tiene una alta capacidad de fijación de nitrógeno, rápida degradación de su hojarasca y un estímulo sobre la diversidad biológica orgánica del suelo. Su presencia permite la recuperación de suelos degradados, convirtiéndola en una leguminosa ideal, ya que aporta proteína como sustratos energéticos, favoreciendo la respuesta del bovino en ganancia de peso y producción de leche, pudiendo garantizar a futuro una mayor productividad y resultados económicos que alienten nuevas perspectivas de producción animal y por ende resultados sociales atribuibles al sector pecuario.

En la búsqueda de recursos forrajeros no convencionales, destaca por su aceptación general, $A$. pintoi o maní forrajero. Este ha causado no solamente un impacto en la línea productiva pecuaria, sino también ornamental, debido a sus cualidades de adaptación en la zona central del Litoral ecuatoriano. Esta leguminosa forrajera posee un hábito de crecimiento postrado, estolonífero y se adapta bien al sombrío (Zelada e Ibrahin, 1996; Andrade y Valentín, 1999), lo cual explica en parte el éxito como cultivo de cobertura. Además, persiste bajo pastoreo, debido a su hábito de crecimiento, la habilidad de sus estolones para enraizar y la alta reserva de semilla en el suelo (Jones, 1993). También presenta un alto potencial para cobertura en cultivos perennes como cítricos (Rincón y Orduz,
2004), plátano (Jhons, 1994) y papaya (Dwyer, 1989), por lo cual se considera una alternativa viable. Los mejores rendimientos se reportan en suelos arenosos con un contenido de materia orgánica superior al 3\% y cuando hay suficiente humedad disponible (Asakawa y Ramírez, 1989). Destaca también el incremento de proteína y digestibilidad, por el aporte de nitrógeno y por una oferta de forraje de mejor calidad para el ganado (Thomas y Asakawa, 1993). Es por estas razones que el presente estudio tuvo como objetivo evaluar la fenología y producción de la planta, así como también determinar la composición química nutricional, digestibilidad in vivo y el desarrollo de modelos matemáticos para determinar la composición química nutritiva y calidad nutricional.

\section{Materiales y Métodos}

\section{Fase 1. Evaluación botánica}

La primera fase experimental se llevó a cabo en la hacienda ESPE San Antonio, ubicada en el km 38.5 carretera Sto. Domingo-Quevedo, parroquia Luz de América, provincia de Santo Domingo de los Tsáchilas. Ubicada geográficamente a 225 metros sobre el nivel del mar, a $0^{\circ} 24^{\prime} 32^{\prime \prime}$ latitud Sur y a $78^{\circ} 18^{\prime} 25^{\prime \prime}$ longitud Oeste. La zona posee un clima húmedo tropical con una textura de suelo franco arenoso. La evaluación botánica se realizó sobre cultivos establecidos de maní forrajero (año 2001) y a los cuales previamente se les realizó un corte de homogeneización. Se establecieron parcelas experimentales de $150 \mathrm{~m}^{2}(10 \mathrm{x} 15 \mathrm{~m}) \mathrm{y}$, sobre estas se realizó un seguimiento de las variables de estudio, medidas a intervalos de 15 días, partiendo a los 30 días de edad; resultando en cuatro fechas de evaluación ( 30 , 45, 60 y 75 días). Las observaciones se realizaron en base a muestras aleatorias en cada parcela con la ayuda de cuadrantes, evaluando altura, cobertura, producción, relación hoja:tallo y análisis proximal de la biomasa. Para la evaluación de altura de planta se seleccionaron 10 plantas al azar mediante el lanzamiento de un cuadrante de $0.25 \mathrm{~m}^{2}$ en cada parcela y se procedió a realizar su medición con una cinta métrica (Bobadilla, 2009). En cuanto a cobertura, por ser una leguminosa rastrera, se estimó a nivel de cobertura basal, utilizando el cuadrante de $1 \mathrm{~m}^{2}$. Esta cobertura fue una estimación basada en el porcentaje del cuadro que estaba cubierto por vegetación y observando suelo desnudo.

La evaluación de producción de materia verde y seca se realizó mediante el corte de la biomasa circunscrito en un cuadrante de $1 \mathrm{~m}^{2}$ en cada parcela, luego se procedió al peso individual de las muestras y se obtuvo el valor medio de las mismas (Bobadilla, 2009). La relación hoja:tallo se evaluó tomando una muestra de $200 \mathrm{~g}$ de material fresco, procedente de las muestras 
de disponibilidad y rendimiento. Posteriormente, se separaron componentes en forma manual (hojas $y$ tallos). Estas muestras se secaron en estufas de aire forzado (Memmert, modelo Tv- 400) hasta obtener un peso constante y pesarse por separado, calculándose el porcentaje de cada fracción en base a materia seca (Bobadilla, 2009).

\section{Fase 2. Digestibilidad in vivo $\mathrm{y}$ análisis químico} nutricional

La segunda fase experimental se llevó a cabo en la Facultad de Ciencias Pecuarias de la ESPOCH, ubicada en el km 1.5 de la carretera Panamericana Sur de la Ciudad de Riobamba. Ubicada geográficamente a 2,780 msnm., a $1^{\circ} 39^{\prime} 58^{\prime \prime}$ de latitud Sur y a 78 $39^{\prime} 33^{\prime \prime}$ de longitud Oeste. Esta zona posee un clima frío ecuatorial húmedo con una textura de suelo franco. En esta fase de la investigación, se utilizaron cuatro ovinos mestizos (Rambouillet x Criollo) de 16 meses de edad y con un promedio de $37 \mathrm{~kg}$ de peso vivo (PV), correspondiendo cada uno de ellos a una unidad experimental. Los animales fueron desparasitados con Albendazole (10 $\mathrm{mg} \mathrm{kg}{ }^{-1}$ de PV) vía oral y se les suministró vitaminas $\mathrm{A}, \mathrm{D}_{3}, \mathrm{E}, \mathrm{B}_{12}$. El alimento que se ofreció a los animales se calculó según las recomendaciones del NRC para ovinos en etapa de mantenimiento (NRC, 1985). El período de adaptación correspondió a seis días, mientras que el período de colecta de datos fue de nueve días. El suministro de la ración se realizó dos veces por día en horarios fijos (08:00 y 16:00 h), de acuerdo con los tratamientos experimentales. Se ofreció agua a voluntad antes de suministrar la nueva ración, se realizó el pesaje y la recolección de alimento sobrante y heces de cada jaula metabólica, la cual tenía un piso de malla para facilitar la recolección, cuyo ángulo de inclinación permitió que el excremento se colectase en un recipiente independiente, evitando el contacto con la orina (Basurto y Tejada, 1992).

Las muestras de alimento sobrante y heces fueron llevadas al laboratorio para el respectivo análisis proximal, donde se determinó: Materia seca (MS), materia orgánica $(\mathrm{MO})$, proteína bruta $(\mathrm{PB} ; \mathrm{N}$ x 6.25), fibra bruta (FB), extracto etéreo (EE) y extracto libre de nitrógeno (ELN) (AOAC, 1990). Estos datos fueron utilizados para la evaluación de digestibilidad y determinación de los nutrientes digestibles totales (NDT). La energía metabolizable (EM) se obtuvó mediante la ecuación propuesta por el NRC (2001):

$\mathrm{EM}=1.01 \times \mathrm{ED}\left(\mathrm{Mcal} \mathrm{Kg}^{-1}\right)-0.45$

Ecuación 1

Para el cálculo de la energía neta de lactancia se estimó con la fórmula propuesta por el NRC (2001):
$\mathrm{EN}_{\mathrm{L}}=0.0245 \times \mathrm{NDT}(\%)-0.12$

Ecuación 2

La evaluación estadística se realizó mediante análisis de varianza con un nivel de significancia del $5 \%(\mathrm{p} \leq 0.05)$. Para la comparación de medias se utilizó la prueba de Tukey. El análisis de correlación, regresión simple y múltiple con ajuste de la tendencia, consideró las variables de respuesta experimental en la fenología a: Altura de planta, peso de hoja, peso de tallos, producción de forraje verde $(\mathrm{FV})$, producción de $\mathrm{MS}$, producción de $\mathrm{PB}$, producción de energía. En la digestibilidad las variables en estudio fueron: Digestibilidad de la MS, digestibilidad de la MO, digestibilidad de la PB, digestibilidad de FB, digestibilidad de EE, digestibilidad de ELN.

Se utilizó un diseño completamente al azar cuyo modelo lineal aditivo es:

$$
(Y i j k)=\mu+\alpha i+\varepsilon i j
$$

Donde: Yijk es el valor de la variable dependiente; $\mu$ es la media general; $\alpha$ i corresponde al efecto debido al tratamiento; y cij al el error experimental.

\section{Resultados y Discusión}

\section{Fenología. Cobertura, altura y peso de hojas del maní forrajero}

En el cuadro 1 se observan los resultados de la evaluación fenológica en cuanto a cobertura (\%), altura $(\mathrm{cm})$, peso de hojas y tallos $\left(\mathrm{kg} \mathrm{m}^{-2}\right)$. En cuanto a cobertura general el maní forrajero constituyó el manto vegetativo en el cultivo primario de palma africana y la cobertura alcanzó alrededor del $97 \%$ en época lluviosa. Este resultado no es marginal si se compara el valor de 98\% de cobertura en cultivo sin asociación y en época lluviosa señalado por Tejos (1997). En cuanto a la altura de planta, estos valores son superiores a los reportados por Tejos (1997), las condiciones climatológicas donde se realizó esta investigación permitieron desarrollar todo su potencial fenológico.

\section{Producción de forraje verde y materia seca del maní forrajero}

El cuadro 2 muestra la producción de forraje verde y materia seca por $\mathrm{m}^{2}$ y en $\mathrm{MS} \mathrm{kg}^{-1}$ por hectárea, destacando que la mayor producción se obtuvo en el corte realizado a los 75 días. La disponibilidad de forraje depende de la fertilidad natural del suelo, precipitación y de la fertilización en el establecimiento y de mantenimiento del maní forrajero. En cuanto al contenido de materia seca se reconocen rangos comprendidos entre: $5,000-9,000 \mathrm{~kg} \mathrm{ha}^{-1}$ reportados por 
Godoy et al.

Cuadro 1. Cobertura, altura, peso de hojas y peso de tallo del $A$. pintoi en diferentes edades de corte

\begin{tabular}{|c|c|c|c|c|c|c|c|c|c|c|}
\hline Variable & 30 días & & 45 días & & 60 días & & 75 días & & $\begin{array}{c}\text { Error } \\
\text { estándar }\end{array}$ & Prob. \\
\hline No. Observaciones & 4 & & 4 & & 4 & & 4 & & & \\
\hline Cobertura $(\%)$ & 98 & $\mathrm{a}$ & 97 & $\mathrm{a}$ & 97 & $\mathrm{a}$ & 98 & & 0.07 & 0.56 \\
\hline Altura (cm) & 18.38 & $\mathrm{~d}$ & 31.92 & $\mathrm{c}$ & 61.06 & $\mathrm{~b}$ & 82.38 & $\mathrm{a}$ & 1.94 & 0.001 \\
\hline Peso de hojas $\left(\mathrm{kg} \mathrm{m}^{-2}\right)$ & 0.58 & d & 1.24 & c & 1.48 & $\mathrm{~b}$ & 2.28 & $\mathrm{a}$ & 0.03 & 0.001 \\
\hline Peso de tallos $\left(\mathrm{kg} \mathrm{m}^{-2}\right)$ & 0.53 & d & 1.25 & $\mathrm{c}$ & 1.38 & b & 2.52 & $\mathrm{a}$ & 0.03 & 0.001 \\
\hline
\end{tabular}

Medias en las misma fila seguidas por la misma letra no presentan diferencias estadísticas (Tukey, $\mathrm{p}<0.05$ ).

Cuadro 2. Poducción de forraje verde y materia seca del $A$. pintoi en diferentes edades de corte

\begin{tabular}{|c|c|c|c|c|c|c|}
\hline Variable & 30 días & 45 días & 60 días & 75 días & $\begin{array}{c}\text { Error } \\
\text { estándar }\end{array}$ & Prob. \\
\hline No. Observaciones & 4 & 4 & 4 & 4 & & \\
\hline Producción de FV, $\left(\mathrm{kg} \mathrm{ha}^{-1}\right)$ & $11,230 \mathrm{c}$ & $24,950 \mathrm{~b}$ & $28,750 \mathrm{~b}$ & $47,730 \mathrm{a}$ & 652.07 & 0.001 \\
\hline Producción MS, $\left(\mathrm{kg} \mathrm{ha}^{-1}\right)$ & $2,469 \quad \mathrm{~d}$ & $6,479 \mathrm{c}$ & $7,084 \mathrm{~b}$ & $12,480 \mathrm{a}$ & 163.70 & 0.001 \\
\hline
\end{tabular}

Medias en las misma fila seguidas por la misma letra no presentan diferencias estadísticas (Tukey, $\mathrm{p}<0.05$ ).

Ferguson et al. (1992). Estos rangos son menores a los reportados en esta investigación que son de 12,480 $\mathrm{kg} \mathrm{ha}^{-1}$.

Esta tendencia se explica por la relación directamente proporcional entre edad de la planta y producción de biomasa, aun cuando la calidad y el valor nutritivo se depriman con la edad del maní forrajero (Ledesma, 1994).

\section{Producción de PB, EM, y $\mathrm{EN}_{\mathrm{L}}$ del maní forrajero}

En el cuadro 3 se observan los valores de proteína bruta, energía metabolizable y energía neta de lactancia por tratamiento. En cuanto a producción de proteína bruta, el mayor aporte se obtuvo en el corte realizado a los 30 días $(\mathrm{p} \leq 0.001)$ siendo $2,730 \mathrm{~PB}\left(\mathrm{~kg} \mathrm{ha}^{-1}\right)$, en segundo lugar fue para el corte a los 75 días con 1,780 de PB $\left(\mathrm{kg} \mathrm{ha}^{-1}\right)$, mientras que el corte de 45 y 60 días alcanzaron una producción de 1,220 y 1,270 PB (kg $\mathrm{ha}^{-1}$ ) respectivamente.

La mayor producción de PB a los 30 días se puede explicar por el hecho que las hojas guardan la mayor reserva de nitrógeno con respecto a los tallos, siendo este factor el que influye en la disminución de PB cuando avanza la edad de la planta (Ledesma, 1994). Esto explicaría la menor concentración en el corte realizado a los 45 días. No obstante, se observa una recuperación gradual en los contenidos de PB en los cortes de 60 y 75 días, debido a que aparecen nuevas yemas en los estolones que son los precursores de nuevas hojas, elevando así el contenido de proteína nuevamente. El maní forrajero, mantiene altos valores nutricionales inclusive a las doce semanas de rebrote. En esas edades, Conejo (2002) reporta valores de $18.74 \%$ de PC.

La mayor producción en cuanto a energía neta de lactancia se obtuvo en el corte realizado a los 75 días, difiriendo con el resto de tratamientos, mientras que

Cuadro 3. Producción de proteína bruta, energía metabolizable y energía neta de lactancia del $A$. pintoi por hectárea en diferentes edades de corte

\begin{tabular}{|c|c|c|c|c|c|c|}
\hline Variable & 30 días & 45 días & 60 días & 75 días & $\begin{array}{c}\text { Error } \\
\text { estándar }\end{array}$ & Prob. \\
\hline Producción PB (kg ha $\left.{ }^{-1}\right)$ & $2,730 \mathrm{a}$ & $1,220 \mathrm{c}$ & $1,270 \mathrm{c}$ & $1,780 \quad b$ & 60.65 & 0.001 \\
\hline Producción de EM $\left(\right.$ Mcal ha-1) $\left.^{-1}\right)$ & $6,630 \mathrm{c}$ & $16,170 \mathrm{~b}$ & $17,070 \mathrm{~b}$ & $28,030 \mathrm{a}$ & 498.01 & 0.001 \\
\hline Producción de $\mathrm{EN}_{\mathrm{L}}\left(\mathrm{Mcal}_{\text {ha-1 }}\right)$ & 3,950 a & $10,300 \mathrm{~b}$ & $11,270 \mathrm{~b}$ & $19,880 \mathrm{a}$ & 367.09 & 0.001 \\
\hline
\end{tabular}

$\mathrm{PB}=$ Proteína bruta, $\mathrm{EM}=$ Energía metabolizable, $\mathrm{EN}_{\mathrm{L}}=$ Energía neta de lactancia

Medias en las misma fila seguidas por la misma letra no presentan diferencias estadísticas (Tukey, $\mathrm{p}<0.05$ ). 
la menor producción se obtuvo en el corte de 30 días. Estos resultados se atribuyen al contenido de azúcares estructurales solubles e insolubles, así conforme avanza la edad de la planta estos al metabolizarse producen más energía. Otro factor importante corresponde a la época del año, ya que además de estar influenciada por la evolución o crecimiento de la planta, las condiciones climáticas tienen un efecto muy importante. Así entonces, los carbohidratos no estructurales (CNES) se producen en las hojas gracias al efecto del sol (fotosíntesis), eso significa que durante los días nublados la síntesis de CNES es más baja (Fernández, 2003).

\section{Composición química nutritiva del maní forrajero en diferentes edades de corte}

El cuadro 4 muestra los resultados de materia orgánica $(\mathrm{MO})$, proteína bruta $(\mathrm{PB})$, fibra bruta $(\mathrm{FB})$, extracto etéreo (EE) y extracto libre de nitrógeno (ELN). No existieron diferencias entre los tratamientos para la variable MO. Estos valores, son similares a los reportados por Duchi (2003) de $91.08 \%$ en maní forrajero. La mayor concentración de PB correspondió al material vegetativo cosechado el día 30 (24.50\%), que disminuyó conforme avanzó el estado fenológico de la planta. Esto es posiblemente debido a la caída de hojas en el secado. Sin embargo, estudios realizados en la Amazonía ecuatoriana con $A$. pintoi (CIAT), citados por Ledesma (1994), indican valores de proteína en un rango del 19.4 a 21\%. Por otro lado, Duchi (2003) reportó un contenido de $\mathrm{PB}$ de $13.4 \%$ en completo estado de maduración y pos floración.

En cuanto a la FB, el menor valor se obtuvo a los 30 días (21.69\%) seguido por la cosecha del día 45 con un valor de $23.76 \%$. Luego sigue el material vegetativo cosechado el día 60 con $26.12 \%$, y a medida que avanza la edad se reporta el mayor valor a los 75 días (29.45\%). Al comparar los resultados de fibra obtenidos en esta investigación con el valor obtenido en la investigación en maní forrajero por Duchi (2003), cuyo valor fue de $29.39 \%$, es posible deducir que mientras avanza la edad de corte de la planta ésta se lignifica y se hace fibrosa (Duchi, 2003).

Analizando el contenido de EE en el maní forrajero no existen diferencias significativas $(p>0.05)$ entre el material vegetativo cosechado el día 30 (6.10\%) y el cosechado el día 45 (5.62\%). Pero a medida que avanza la edad disminuye la concentración de EE con valores de $4.66 \%$ en el día 60 , el cual si presenta diferencias significativas $(\mathrm{p}>0.05)$ con el corte de 30 días, observando que el menor valor fue para el corte realizado a los 75 días con 3.41\%. Por su parte, Duchi (2003) reportó a los 75 días de cosecha valores de $3.19 \%$, estas diferencias se deberían a la presencia de cutinas.

En cuanto al ELN, el mejor valor se observó en el material vegetativo cosechado el día 75 (42.54\%) y el

Cuadro 4. Composición química del $A$. pintoi en diferentes edades de corte

\begin{tabular}{|c|c|c|c|c|c|c|}
\hline Variable & 30 días & 45 días & 60 días & 75 días & $\begin{array}{l}\text { Error } \\
\text { estándar }\end{array}$ & Prob. \\
\hline No. de observaciones & 4 & 4 & 4 & 4 & & \\
\hline Materia orgánica, \% & $87.79 \mathrm{a}$ & $88.97 \mathrm{a}$ & $89.88 \mathrm{a}$ & $90.05 \mathrm{a}$ & 0.154 & 0.59 \\
\hline Proteína Bruta, \% & $24.50 \mathrm{a}$ & $21.02 \mathrm{~b}$ & $17.84 \mathrm{c}$ & $14.65 \mathrm{~d}$ & 0.193 & 0.001 \\
\hline Fibra Bruta, \% & $21.69 \mathrm{a}$ & $23.76 \mathrm{~b}$ & $26.12 \mathrm{c}$ & $29.45 \mathrm{~d}$ & 0.194 & 0.001 \\
\hline Extracto etéreo, $\%$ & $6.10 \mathrm{a}$ & $5.62 \mathrm{a}$ & $4.66 \mathrm{~b}$ & $3.41 \mathrm{c}$ & 0.073 & 0.001 \\
\hline Extracto libre de nitrógeno, \% & $37.50 \mathrm{c}$ & $40.57 \quad b$ & $42.26 \mathrm{a}$ & $42.54 \mathrm{a}$ & 0.141 & 0.001 \\
\hline
\end{tabular}

Medias en las misma fila seguidas por la misma letra no presentan diferencias estadísticas (Tukey, p<0.05).

menor valor fue en el corte a los 30 días (37.50\%). Esto contrasta con los resultados reportados por Duchi (2003) $61.81 \%$. Las diferencias podrían deberse posiblemente a la madurez de la planta la cual no fue determinada en el estudio de Duchi (2003).

\section{Digestibilidad in vivo}

La evaluación de digestibilidad in vivo se reporta en el cuadro 5, el que resume los valores de digestibilidad de la materia seca (DMS), materia orgánica (DMO), proteína cruda (DCP), extracto etéreo (DEE) y extracto libre de nitrógeno (DELN), valores que a su vez son útiles para el cálculo y estimación de los nutrientes digestibles totales (NDT).

\section{Digestibilidad de la Materia Seca}

La digestibilidad in vivo de la MS registró diferencias significativas $(\mathrm{p}=0.037)$ entre las medias de los diferentes tratamientos. Así el material cosechado el día 30 obtuvo mayor digestibilidad (66.42\%) en comparación al material cosechado el día 75, el que registro la menor digestibilidad (57.84\%). Estos valores 
Godoy et al.

Cuadro 5. Digestibilidad aparente del $\boldsymbol{A}$. pintoi en diferentes edades de corte

\begin{tabular}{lcccccc}
\hline Variable & 30 días & 45 días & 60 días & $\mathbf{7 5}$ días & $\begin{array}{c}\text { Error } \\
\text { estándar }\end{array}$ & Prob. \\
\hline No. de observaciones & 4 & 4 & 4 & 4 & & \\
Digestibilidad MS, \% & $66.42 \mathrm{a}$ & $62.6 \mathrm{ab}$ & $60.2 \mathrm{~b}$ & $57.8 \mathrm{~b}$ & 1.86 & 0.04 \\
Digestibilidad MO, \% & $68.65 \mathrm{a}$ & $64.8 \mathrm{ab}$ & $62.1 \mathrm{bc}$ & $59.5 \mathrm{c}$ & 1.77 & 0.02 \\
Digestibilidad PC, \% & $78.03 \mathrm{a}$ & $74.7 \mathrm{a}$ & $69.3 \mathrm{~b}$ & $68.6 \mathrm{~b}$ & 1.24 & 0.00 \\
Digestibilidad FB, \% & $43.38 \mathrm{a}$ & $39.2 \mathrm{ab}$ & $33.7 \mathrm{bc}$ & $28.7 \mathrm{c}$ & 3.09 & 0.03 \\
Digestibilidad EE, \% & $58.49 \mathrm{a}$ & $54.9 \mathrm{ab}$ & $49.6 \mathrm{ab}$ & $47.3 \mathrm{~b}$ & 2.55 & 0.06 \\
Digestibilidad ELN, \% & $80.31 \mathrm{a}$ & $76.4 \mathrm{a}$ & $76.6 \mathrm{a}$ & $78.7 \mathrm{a}$ & 1.64 & 0.33 \\
\hline
\end{tabular}

son menores a los señalados por Pérez (2000) quien reportó una DMS superior al 70\%. Por otra parte, Duchi (2003) reporta valores de DMS superiores al 70 y $74 \%$, respectivamente. Estos altos valores pueden deberse a diferentes factores como la relación entre tallos y hojas, el efecto animal o preferentemente la depresión de consumo que tienen los ovinos tropicales frente a los ovinos europeos.

\section{Digestibilidad de la Materia Orgánica}

El mayor coeficiente de digestibilidad in vivo de materia orgánica de los tratamientos evaluados fue el material cosechado el día 30 con $(68.65 \%)$, seguido por el cosechado el día 45 (64.76\%), luego el material cosechado el día 60 (62.05\%), y por último el cosechado el día 75 (59.50\%), siendo este último el que presentó el menor valor de digestibilidad. Debemos considerar que estos valores son bajos comparados con lo reportado por Duchi (2003) de $75.90 \%$. Esto podría deberse al estado fenológico y época de corte.

\section{Digestibilidad de la Proteína}

Los valores de coeficiente de digestibilidad de la proteína cruda cosechados a los 30 y 45 días no difieren entre si ( $p>0.05)$, pero ambos son mejores respecto a los cosechados en los 60 y 75 días, los que a su vez tampoco difieren entre ellos $(\mathrm{p}>0.05)$. En tal sentido, estudios realizados por otros autores reportan coeficientes de digestibilidad aparente de la proteína de $80.4 \%$ (Duchi, 2003), mientras que Tejos (1997) reportó rangos de 62 a $67 \%$. Esta cualidad permite considerar al A. pintoi una alternativa para suplir proteína degradable en el rumen y consecuentemente proteína metabolizable en aquellos sistemas en los cuales la demanda es alta, como ocurre en vacas altas productoras (Villareal et al., 2005).

\section{Digestibilidad de la Fibra}

Los valores reportados del material cosechado a los 30 y 45 días no difieren estadísticamente, siendo seguido por el material cosechado el día 60 y por el material cosechado el día 75. Estos valores puede explicarse porque en las leguminosas el contenido de la pared celular es menor y por lo tanto sus contenidos celulares son mayores. El maní forrajero no es la excepción presentando el $A$. pintoi CIAT 18744A (Conejo, 2002) valores de 34 a $40 \%$ de fibra detergente neutro (FDN) correspondiendo a contenidos celulares de 66 y $60 \%$ respectivamente.

\section{Digestibilidad del Extracto Etéreo (DEE)}

Analizando la digestibilidad in vivo del extracto etéreo, en los coeficientes de digestibilidad se observa una disminución en la medida que aumenta las fechas de corte (Cuadro 5). Lamentablemente, el extracto etéreo no refleja el verdadero valor nutricional de la fracción lipídica de los alimentos. En algunos alimentos, como los forrajes, una parte importante del extracto etéreo está compuesto por sustancias insaponificables (ceras, terpenos, etc.) de nulo valor energético para los animales (Palmquist y Jenkins, 2003).

\section{Digestibilidad del Extracto libre de nitrógeno}

En la digestibilidad del ELN se pudo determinar un mayor valor en el material cosechado a los 30 días de edad con $80.31 \%$, aún cuando no difiere estadísticamente con ninguno de los cosechados a los 45, 60 y 75 días. Por el hecho de comprender teóricamente, aquellos carbohidratos solubles y de elevada digestibilidad como los azúcares y el almidón, el ELN debería ser altamente digestible sin embargo, al contener en los pastos parte de la hemicelulosa presente en la planta y parte de la lignina, su digestibilidad es usualmente más baja de la esperada.

Es así que la mayor digestibilidad de la FDN, en relación con la digestibilidad de la fibra detergente ácida (FDA), es atribuible a la mayor presencia de lignina en la FDA. Para el heno A. pintoi, la lignina fue parte del 21.3 y el $31.3 \%$ de FDN y FDA, respectivamente. Este hecho, es decir, la ausencia de lignina, puede explicar la superioridad y digestibilidad de hemicelulosa (HCEL) 
en comparación con otras fracciones de la fibra (Ladeira et al., 2002).

\section{Nutrientes Digestibles Totales (NDT)}

El corte del material vegetativo realizado a los 30 días fue el mejor respecto a los realizados a los 45 , 60 y 75 días (Cuadro 6). Ésto se debe a que el corte de 30 días presenta una superioridad tanto en lo que se refiere a composición química como el coeficiente de digestibilidad. En investigaciones con asociaciones forrajeras el valor promedio en $A$. pintoi fue $51.1 \%$ (Sánchez et al., 2000) que es menor a los valores obtenidos en ésta investigación.

\section{Energía Digestible (ED)}

El contenido medio de energía digestible registrado en el corte realizado a los 30 días fue el mayor con 2.93 Mcal kg-1, seguido del corte realizado a los a los 45 días (2.77 $\left.\mathrm{Mcal} \mathrm{kg}^{-1}\right)$. El corte realizado a los 60 días presentó un aporte de $2.59 \mathrm{Mcal} \mathrm{kg}^{-1} \mathrm{y}$ el menor aporte se obtuvo con el corte realizado a los 75 días $(2.45 \mathrm{Mcal}$ $\mathrm{kg}^{-1}$ ). Conejo (2002) en investigaciones realizadas en $A$. pintoi $18744 \mathrm{~A}$, cuantifica valores de $2.51 \mathrm{Mcal} \mathrm{kg}^{-1} \mathrm{MS}$.

\section{Energía Metabolizable (EM)}

Las medias registradas fueron significativas en el corte realizado a los 30 días en relación con los cortes realizados a los 60 y 75 días, ya que el corte realizado a los 30 días presenta el mayor aporte de EM (2.38 Mcal $\mathrm{kg}^{-1}$ ), que es superior al corte realizado a los 45 días (2.25 Mcal $\mathrm{kg}^{-1}$ ), seguido por el corte realizado a los 60 días $\left(2.10 \mathrm{Mcal} \mathrm{kg}^{-1}\right)$, y por último al corte realizado a los 75 días $\left(1.98 \mathrm{Mcal} \mathrm{kg}^{-1}\right)$. Investigaciones realizadas con maní forrajero en asociación con gramíneas determinan valores de energía metabolizable inferiores a los obtenidos en ésta investigación (1.83 Mcal kg de MS), atribuyendo estos valores a la altura tan baja que fueron tomadas las muestras que originaron ésta investigación (Sánchez et al., 2000).

\section{Energía Neta de Lactancia $\left(\mathrm{EN}_{\mathrm{L}}\right)$}

El corte realizado a los 30 días aportó una mayor cantidad de energía neta para la producción láctea, alcanzando 1.51 Mcal $\mathrm{kg}^{-1}$, seguido por el corte realizado a los 45 días (1.43 $\left.\mathrm{Mcal} \mathrm{kg}^{-1}\right)$, luego el corte realizado a los 60 días $\left(1.32 \mathrm{Mcal} \mathrm{kg}^{-1}\right)$, y por último el corte realizado a los 75 días, el cual presentó el menor aporte energético con 1.24 Mcal kg-1 (Cuadro 6). A las 12 semanas de rebrote de $A$. pintoi 18744 A en esas edades, Conejo (2002) reporta un contenido de 1.27 Mcal kg-1 de MS.

Globalizando las respuestas determinadas del aporte de energía tanto digestible como metabolizable para la producción láctea, se puede considerar que el material vegetativo cosechado a los 30 días es el que presentó las mejores características. Además, presentó mejores características de digestibilidad de los diferentes nutrientes que lo conforman.

\section{Energía Neta de Ganancia $\left(\mathrm{EN}_{\mathrm{G}}\right)$}

$\mathrm{El}$ mayor valor de $\mathrm{EN}_{\mathrm{G}}$ se obtuvo con el corte realizado a los 30 días, el cual no tuvo diferencias con el corte del día 45, pero si con los cortes realizados en los días 60 y 75 (Cuadro 6).

\section{Metabolicidad}

En cuanto a la metabolicidad, el mejor tratamiento fue aquel cosechado a los 30 días. La menor metabolicidad se obtuvo con el material cosechado al día 75, el que fue distinto con los cortes de 45 y 60 días

Cuadro 6. Contenido de NDT (\%) y aporte de energía (Mcal $\mathrm{kg}^{-1}$ ) MS del A. pintoi en diferentes edades de corte

\begin{tabular}{lcccccc}
\hline Variables & 30 días & $\mathbf{4 5}$ días & $\mathbf{6 0}$ días & $\mathbf{7 5}$ días & $\begin{array}{c}\text { Error } \\
\text { estandar }\end{array}$ & Prob. \\
\hline No. de observaciones & 4 & 4 & 4 & 4 & & \\
NDT (\%) & $66.67 \mathrm{a}$ & $62.97 \mathrm{ab}$ & $58.73 \mathrm{~b}$ & $55.59 \mathrm{~b}$ & 1.94 & 0.01 \\
EB $\left(\mathrm{Mcal} \mathrm{kg}^{-1}\right) \mathrm{MS}$ & $4.43 \mathrm{a}$ & $4.42 \mathrm{a}$ & $4.4 \mathrm{a}$ & $4.34 \mathrm{a}$ & 0.04 & 0.835 \\
$\mathrm{ED}\left(\mathrm{Mcal} \mathrm{kg}^{-1}\right) \mathrm{MS}$ & $2.93 \mathrm{a}$ & $2.77 \mathrm{ab}$ & $2.59 \mathrm{~b}$ & $2.45 \mathrm{~b}$ & 0.09 & 0.008 \\
$\mathrm{EM}\left(\mathrm{Mcal} \mathrm{kg}^{-1}\right) \mathrm{MS}$ & $2.38 \mathrm{a}$ & $2.25 \mathrm{ab}$ & $2.1 \mathrm{~b}$ & $1.98 \mathrm{~b}$ & 0.07 & 0.008 \\
$\mathrm{EN}_{\mathrm{L}}\left(\mathrm{Mcal} \mathrm{kg}^{-1}\right) \mathrm{MS}$ & $1.51 \mathrm{a}$ & $1.43 \mathrm{ab}$ & $1.32 \mathrm{~b}$ & $1.24 \mathrm{~b}$ & 0.05 & 0.008 \\
EN $_{\mathrm{G}}\left(\mathrm{Mcal} \mathrm{kg}^{-1}\right) \mathrm{MS}$ & $1.32 \mathrm{a}$ & $1.25 \mathrm{ab}$ & $1.16 \mathrm{~b}$ & $1.1 \mathrm{~b}$ & 0.03 & 0.008 \\
Metabolicidad (q) & $53.72 \mathrm{a}$ & $50.9 \mathrm{ab}$ & $47.72 \mathrm{~b}$ & $45.62 \mathrm{~b}$ & 0.01 & 0.037 \\
\hline
\end{tabular}

Medias en las misma fila seguidas por la misma letra no presentan diferencias estadísticas (Tukey, $\mathrm{p}<0.05$ ). 
Godoy et al.

$(\mathrm{p}<0.05)$.

En esta investigación se estudió el grado de asociación entre variables de producción, composición química, digestibilidad y energía. Además, se obtuvieron ecuaciones de estimación para predecir estas variables, las que se presentan en el cuadro 7 .

Cuadro 7. Ecuaciones de predicción de variables de producción, digestibilidad y energía del $A$. pintoi en diferentes edades de corte

\begin{tabular}{|c|c|c|c|}
\hline Variable & Ecuación & $\mathbf{r}^{2}$ & Prob. \\
\hline Producción Forraje verde & PDN FV $\left(\mathrm{m}^{-2}\right)=0.0755($ edad de corte $)-1.1493$ & 0.94 & 0.001 \\
\hline Producción Materia Seca & PDN MS $\left(\mathrm{m}^{-2}\right)=0.0202$ (edad de corte) -0.3535 & 0.92 & 0.001 \\
\hline Producción EM & $\operatorname{EM}\left(\right.$ Mcal m$\left.^{-2}\right)=0.044($ edad de corte $)+0.603$ & 0.91 & 0.001 \\
\hline Producción $\mathrm{EN}_{\mathrm{L}}$ & $\mathrm{EN}_{\mathrm{L}}\left(\right.$ Mcal m$\left.{ }^{-2}\right)=0.0325($ edad de corte $)-0.5713$ & 0.92 & 0.001 \\
\hline Producción de Leche & PDN Leche $\left(\mathrm{L} \mathrm{ha}^{-1}\right)=354.71\left(\mathrm{EM} \mathrm{ha}^{-1}\right)-4,880.8$ & 0.91 & 0.001 \\
\hline Producción de Leche & PDN Leche $\left(\mathrm{L} \mathrm{ha}^{-1}\right)=462.05\left(\mathrm{ENL} \mathrm{ha}^{-1}\right)-9,771.9$ & 0.85 & 0.001 \\
\hline Digestibilidad MS & DMS $(\%)=-0.1879($ edad de corte $)+71.643$ & 0.49 & 0.001 \\
\hline Digestibilidad MO & $\operatorname{DMO}(\%)=-0.2011($ edad de corte $)+74.295$ & 0.55 & 0.001 \\
\hline NDT & $\operatorname{NDT}(\%)=-0.2497($ edad de corte $)+74.104$ & 0.65 & 0.001 \\
\hline Contenido EM & $\mathrm{EM}\left(\mathrm{Mcal} \mathrm{kg}{ }^{-1}\right) \mathrm{MS}=0.0088($ edad de corte $)+2.9121$ & 0.59 & 0.001 \\
\hline Contenido $\mathrm{EN}_{\mathrm{L}}$ & $\mathrm{EN}_{\mathrm{L}}\left(\mathrm{Mcal} \mathrm{kg}{ }^{-1}\right) \mathrm{MS}=-0.00611(\mathrm{edad}$ de corte $)+1.6956$ & 0.65 & 0.001 \\
\hline Contenido $\mathrm{EN}_{\mathrm{G}}$ & $\mathrm{EN}_{\mathrm{G}}\left(\mathrm{Mcal} \mathrm{kg}{ }^{-1}\right) \mathrm{MS}=-0.005($ edad de corte $)+1.4705$ & 0.65 & 0.001 \\
\hline Digestibilidad MO & $\operatorname{DMO}(\%)=1.1253(\% \mathrm{~PB})+43.203$ & 0.54 & 0.001 \\
\hline Digestibilidad MO & $\mathrm{DMO}(\%)=-1.1155(\% \mathrm{FB})+91.909$ & 0.54 & 0.001 \\
\hline NDT & $\operatorname{NDT}(\%)=1.3896(\% \mathrm{~PB})+35.633$ & 0.64 & 0.001 \\
\hline NDT & $\operatorname{NDT}(\%)=-1.3786(\%$ FB $)+95.806$ & 0.63 & 0.001 \\
\hline Contenido EM & $\mathrm{EM}\left(\mathrm{Mcal} \mathrm{kg}{ }^{-1}\right) \mathrm{MS}=0.0496(\% \mathrm{~PB})+1.2726$ & 0.63 & 0.001 \\
\hline Contenido EM & $\mathrm{EM}\left(\mathrm{Mcal} \mathrm{kg}^{-1}\right) \mathrm{MS} .=-0.0492(\% \mathrm{FB})+3.4215$ & 0.63 & 0.001 \\
\hline Contenido $\mathrm{EN}_{\mathrm{L}}$ & $\left.\mathrm{EN}_{\mathrm{L}}(\mathrm{Mcal} \mathrm{kg})^{-1}\right) \mathrm{MS}=0.034(\% \mathrm{~PB})+0.753$ & 0.63 & 0.001 \\
\hline Contenido $\mathrm{EN}_{\mathrm{L}}$ & $\mathrm{EN}_{\mathrm{L}}\left(\mathrm{Mcal} \mathrm{kg}^{-1}\right) \mathrm{MS}=-0.0338(\% \mathrm{FB})+2.2272$ & 0.63 & 0.001 \\
\hline Contenido $\mathrm{EN}_{\mathrm{G}}$ & $\mathrm{EN}_{\mathrm{G}}\left(\mathrm{Mcal} \mathrm{kg}^{-1}\right) \mathrm{MS}=0.0276(\% \mathrm{~PB})+0.7057$ & 0.63 & 0.001 \\
\hline Contenido $\mathrm{EN}_{\mathrm{G}}$ & $\left.\mathrm{EN}_{\mathrm{G}}(\mathrm{Mcal} \mathrm{kg})^{-1}\right) \mathrm{MS}=-0.0274(\% \mathrm{FB})+1.9022$ & 0.63 & 0.001 \\
\hline
\end{tabular}

\section{CONCLUSIONES}

$\mathrm{E}^{1}$ 1 estudio permitió caracterizar la fenología de $A$. pinto $i$ bajo condiciones tropicales destacando que el corte de 75 días registró la mayor producción de forraje verde y materia seca. Asimismo, es posible concluir que cortes tardíos tienen un impacto en la calidad nutricional al que indudablemente afectará la respuesta productiva del animal porque la planta se lignifica y se hace más fibrosa.

En cuanto a la composición química, el contenido de proteína bruta disminuye con la edad de corte, así entonces a menor estado vegetativo de la planta mayor contenido de proteína bruta y menor contenido de fibra bruta.
La mayor digestibilidad de MS, MO, PB, FB, EE, ELN, se obtiene a edades tempranas, lo que se explica por la menor lignificación de la pared celular, lo que permite mayor facilidad de digestión.

El contenido energético es inversamente proporcional a la edad de corte, lo que se debe a la menor presencia de almidones y mayor contenido de carbohidratos estructurales en las plantas maduras.

La edad de corte resultó ser un muy buen predictor de calidad, productividad y digestibilidad del maní forrajero. Los modelos obtenidos servirán de base aplicativa en los actuales sistemas de producción animal pudiendo formular raciones en la que la dieta base sea el maní forrajero. 


\section{LiTERATURA CITADA}

Acosta, M. 1997. Plantas indígenas para forrajicultura tropandina. (en línea). Consultado 25 Jun. 2012. Disponible en: www.accefyn.org.co/revista/56/57 -97.pdf

Andrade, C. M. S. y J. F. Valentín. 1999. Adaptacao, produtividade e persistencia de Arachis pintoi submetido a diferentes niveis de sombreamento. Rev. Bras. Zoot. 28(3):439-445.

AOAC. 1990. Official methods of analysis of the Association of Official Analytical Chemists. 15th edition. Washington, DC. 1298 p.

Asakawa, N., y C. A. Ramírez. 1989. Metodología para la inoculación y siembra de Arachis pintoi. Pasturas Tropicales. 11(1):24-26.

Basurto, R. e I. Tejada de Hernández. 1992. Digestibilidad aparente de la pulpa deshidratada de limón. Comparación de métodos para estimarla. Téc. Pec. Méx. 30(1):13-22

Bobadilla, A. 2009. Manual de prácticas de producción y aprovechamiento de forrajes. (en línea). Consultado 25 Jun. 2012. Disponible en: www. fmvz.unam.mx/../PRODUCCIONFORRAJES.doc

Conejo, E. A. 2002. Producción de biomasa y valor nutritivo de la línea de maní forrajero CIAT 18744A en la zona central húmeda de Costa Rica. Tesis Ing. Agr., Facultad de Ciencias Agroalimentarias, Universidad de Costa Rica. 69 p.

Duchi, N. 2003. Valoración nutritiva de subproductos no tradicionales para la alimentación de rumiantes. ESPOCH - PRONSA - IQ-CV-024. Riobamba, Ecuador

Dwyer, G. T. 1989. Pinto's peanut: a ground cover for orchards. Qld, Agric. J. (May-June):153-154

Ferguson, J. E., C. I. Cardoso y M. S. Sánchez. 1992. Avances y Perspectivas en la producción de Semillas de Arachis pintoi". Pasturas Tropicales. CIAT.(2):14:14-22.

Fernández, A. 2003. El efecto de los azúcares solubles sobre la ganancia de peso y su relación con el manejo de los verdeos de invierno. Desafío 21, 9(20):34-37.

Jhons, G. G. 1994. Effect of Arachis pintoi groundcover on performance of bananas northern. New South Wales, Aust. J. Exp. Agric. 34:1197-1204

Jones, R. M. 1993. Persistence of Arachis pintoi cv. Amarillo three soil types of Samford, Queensland. Trop. Grassi. 27:11-15

Ladeira, M. M., N. M. Rodriguez, L. C. Goncalves, E. Silva, S. C. Brito, L. A. Sá. 2002. Avaliação do Feno de Arachis pintoi Utilizando o Ensaio de Digestibilidade in vivo* Rev. Bras. Zootecnia. Vol. 31 No. 6.
Ledesma, J. 1994. Evaluación bajo pastoreo del consumo de Arachis pintoi Krap. Et Greg. y Pueraria phaseoloides Rexb. y asociados con Panicum máximum Jacq. Tesis de grado previo a la obtención de Ing. Agr. Quito. Ecuador. 24-27 p

NRC (National Research Council). 1985. Nutrient Requirements National Academy Press (on line). Researched 8 Ago. 2005. Available en: http://www. nap.edu/openbook.php?isbn=0309035961

NRC (National Research Council). 2001. Nutrient Requirements of Dairy Cattle, Seventh Revised. Ed. Washington, D.C. National Academy Press. $381 \mathrm{p}$.

Palmquist D. L., T. C. Jenkins. 2003. Challenges with fats and fatty acids methods. J. Anim. Sci. 81:3250-3254.

Pérez N. B. 2000. Arachis Pintoi una historia de éxito. (en línea). Consultado 7 Dic. 2005. Disponible en: http://laboratoriosprovet.com/inftecnica/PA STOS \%20Y\%20FORRAJES/ARACHIS \%20 PINTOI.asp

Rincón, A.C. y J.O. Orduz. 2004. Usos alternativos de Arachis pintoi: Ecotipos promisorios como cobertura de suelos en el cultivo de cítricos. Pasturas Tropicales. 26(2):2-8.

Sánchez, J., M. Villarreal, H. Soto. 2000. Caracterización nutricional de los componentes forrajeros de cuatro asociaciones gramíneas Arachis pintoi. Nutrición Animal Tropical, Vol. 6, No 1 .

SICA/MAG. 2002. III Censo Agropecuario. (en línea). Consultado 7 Dic. 2005. Disponible en: http:// www.sica.gov.ec/censo/

Tejos, R. 1997. Características, manejo y perspectivas del maní forrajero Arachis pintoi Kraspovickas e Gregory en el llano venezolano. (en línea). Venezuela Bovina, ed. No. 54. 58 p. Consultado 7 Dic. 2005. Disponible en: http://www.Venezuela bovina.com

Thomas, R. y N. Asakawa. 1993. Descomposition of leaf litter from tropical forage grasses and legumes. Soil Biol. Biochem 25:1351-1361.

Valls, J. F. M. y C. E. Simpson. 1995. Taxonomía, distribución natural y atributos de Arachis. En: P. C. Kerridge (ed.). Biología y agronomía de especies forrajeras de Arachis. Centro Internacional de Agricultura Tropical (CIAT), Cali, Colombia. Publicación No. 245. p. 1-20.

Villareal, M. R. C. Conchran, L. Villalobos, A. Rojas Bourrilow, R. Rodríguez, T. A. Wicherssham. 2005. Dry matter yield and crude protein and rumen degradable protein concentractions of three Arachis pintoi ecoiypes and diferents stages of regrowth in the humids tropics. Grass and Forage Science 60:237-243. 
Godoy et al.

Zelada, E. y M. Ibrahin. 1996. Efectos de diferentes niveles de sombra sobre la morfología, fenología y nodulación del Arachis pintoi. Memorias XVIII reunión Latinoamericana de Rhizobiología (eds J, Pijnebord, D. Ruiz, V. Sila), ALAR Santa Cruz Bolivia. 